\title{
5
}

\section{Relationships Among Speech Perception and Language Measures in Hard-of-Hearing Children}

\author{
Peter J. Blamey, Julia Z. Sarant, E Louise E. Paatsch
}

Spoken communication has long been seen as being at the core of human society and human cognitive development. As a consequence, the development of spoken language is usually seen to be of central importance to all children, including deaf and hard-of-hearing children. The question "How do children learn to make sense from the complex acoustic speech signal?" has interested psychologists, physiologists, and linguists over many years: Brown (1973), Cutler and Swinney (1987), Eimas and Corbit (1973), Jusczyk (1993), Klatt (1979), Mehler et al. (1988), Vihman (1996), and Werker and Tees (1984), to name but a few. How hard-of-hearing children learn to make sense from the complex acoustic speech signal is an even more complex question, and less is known about the answer. A recent summary from the present authors' perspective may be found in Blamey (2002). The main points are as follows:

- Children fall into three hearing categories for the purpose of spoken language development: hearing with pure-tone average (PTA) thresholds up to $25 \mathrm{~dB}$ HL (hearing level), hard-of-hearing with PTA thresholds between 25 and $90 \mathrm{~dB}$ HL; and deaf with PTA thresholds greater than $90 \mathrm{~dB}$ HL.

- Cochlear implants can move a child from the deaf group to the

1. hard-of-hearing group.

- Within the hard-of-hearing category, most children develop spoken language in a similar systematic fashion but usually delayed relative to the hearing group. 
- Within the hard-of-hearing group, the degree of hearing loss

w. has surprisingly low correlation with the rate of spoken länguage development or delay.

- Reduced auditory experience is clearly at the root of spoken language delay for hard-of-hearing children, and therefore early intervention is an important (some would say critical) factor in maximizing the child's exposure to spoken language.

Despite our incomplete psycholinguistic knowledge of how children learn spoken language, families and teachers of hard-of-hearing children are faced with the very real problem of how to help them achieve spoken language at a normal rate. This chapter describes some of the authors' research to evaluate potential solutions to this problem. We have taken a pragmatic experimental approach rather than a theoretical one, going where the data have taken us rather than following any particular learning model, with one exception. We chose not to include children who use sign or other manual communication in our research, because we were less familiar with these communication methods. The inclusion of sign would also complicate both the assessment of spoken language and the description of the development process.

The basis of a pragmatic comparative approach to spoken language development is quantitative assessment using reliable, repeatable, normalized measures. Speech perception tests are commonly used by audiologists to assess the benefits derived from hearing aids and cochlear implants. Language measures are often used by speech pathologists and teachers to assess the spoken language development and educational needs or progress of hard-of-hearing children. If we are to assess the contributions of different devices and habilitation methods to language development, their effects need to be expressed in a common metric, hence the title of this chapter.

Speech perception and language measures are clearly related, and yet the results are often interpreted in different ways, according to the circumstances under which the test was conducted. Some comprehensive language measures (e.g., the Clinical Evaluation of Language Fundamentals [CELF]; Wiig, Secord, \& Semel, 1992; Semel, Wiig, \& Secord, 1995) include a speech perception subtest. It is ironic that the Recalling Sentences in Context subtest of the CELF is classified as an expressive language measure, while an identical measure could be used as a measure of speech perception in an audiological evaluation. Both points of view can be justified if certain prior conditions are satisfied-that is, the test is a test of perception if the person being tested has perfect adult language, and a test of expressive language if the person being tested has perfect hearing and perfect receptive language. Unfortunately, none of these conditions is likely to be satisfied for hard-of-hearing children, and so the interpretation is difficult to say 
the least. To interpret the results correctly, one needs to know the effects of both hearing loss and limited language capabilities on the measures being used. If the effects of the variables can be separated, one has a very effective and generally applicable tool for studying language development and the benefits of cochlear implants, hearing aids, and various educational strategies for hard-of-hearing children.

In this chapter we discuss two approaches to studying the relationships between speech perception and language measures: an empirical multivariate regression analysis of longitudinal data from a large group of children (Blamey et al., 2001), and a more analytical approach based on a few simple assumptions about the ways in which hearing, lexical knowledge, and speech production abilities might be combined to yield a predicted score for a speech perception test (Paatsch, Blamey, Sarant, Martin, \& Bow, 2004). The two approaches lead to some strong hypotheses about the effectiveness of cochlear implants compared to hearing aids for some groups of children, and about the effectiveness of language-based habilitation on the speech perception of children with hearing loss. These hypotheses are being tested using clinical and experimental data.

\section{RELATIONSHIPS AMONG SPEECH PERCEPTION, PRODUCTION, LANGUAGE, HEARING LOSS, AND AGE}

In this first study, the relationships between speech perception, spoken language, hearing, and age-related measures were explored for a group of 87 children who were assessed annually in a longitudinal study (Blamey et al., 2001). Table 5.1 gives details of the children studied. Some of the children used hearing aids (HA group), and some used

Table 5-1: Details of Children Studied

\begin{tabular}{lcc}
\hline & $\begin{array}{l}\text { Cochlear } \\
\text { Implant Users }\end{array}$ & Hearing Aid Users \\
\hline Number of children & 47 & 40 \\
Number with congenital loss & 39 & 34 \\
Total number of evaluations & 80 & 72 \\
Hearing loss (dB HL) & $106(11)[77-125]$ & $78(17)[40-103]$ \\
Onset of hearing loss (years) & $0.3(0.7)[0-3.4]$ & $0.4(1.1)[0-4.6]$ \\
Age at device fitting (years) & $3.5(1.5)[1.2-8.2]$ & Not known \\
Age at evaluation (years) & $7.7(2.0)[4.3-13.0]$ & $9.0(2.4)[4.5-13.5]$ \\
Duration of deafness (years) & $3.2(1.5)[0.5-8.2]$ & Not applicable \\
Auditory experience (years) & $4.2(2.0)[0.9-9.2]$ & $8.5(2.5)[0.6-13.5]$ \\
\hline
\end{tabular}

For rows, data are mean (standard deviation) [range] for each group of children. From Blamey et al. (2001) Copyright by the American Speech-Language-Hearing Association. Reprinted with permission. 
cochlear implants (CI group). All were enrolled in oral/aural primaryschool programs for children with hearing loss in Victoria and New South Wales, Australia. At the time the data were analyzed, some of the children had been assessed once, some twice, and some three times.

Data for a wide variety of perception, production, and language measures were collected, but only an abbreviated set are reported here for clarity and consistency with the second study reported below: The speech perception test scores are for the Consonant-NucleusConsonant (CNC) word test (Peterson \& Lehiste, 1962), the speech production measure is percentage consonants correct (PCC; Shriberg, Austin, Lewis, Sweeny, \& Wilson, 1997), and the language measure is the Peabody Picture Vocabulary test (PPVT; Dunn \& Dunn, 1981, 1997). The CNC word test consists of lists of 50 monosyllabic words each composed of an initial consonant, a vowel, and a final consonant. They were presented to the children with live voice at a level of $65 \mathrm{~dB}$ SPL (sound pressure level) at a distance of $1 \mathrm{~m}$, either in the auditory alone (A) condition or auditory visual (AV) condition with lipreading and hearing together. The children were required to respond verbally, and a word was counted as correct if every phoneme in the word was correctly produced. The PCC measure was derived from spontaneous conversations that were videotaped and later transcribed by a linguist experienced in the transcription of similar speech samples from hardof-hearing children. A narrow transcription method was used, and a consonant was considered correct only if it matched the target phoneme in the word and no diacritic marks were used in its transcription. Only singleton consonants (excluding consonants in clusters) were counted in the calculation of the PCC. The PPVT consists of single words that were presented in the AV condition, and the child was required to select one of four pictures that best represented the meaning of the word. Each score for the PPVT was converted to an equivalent age, the age at which typical hearing children score the same on average as the child whose language is being assessed.

Figures 5.1 and 5.2 show the $\mathrm{CNC}$ word scores in the A condition and the PPVT equivalent ages, respectively. Both figures show improvements in scores over time, and very little difference in the level of the scores or the trends for the $\mathrm{CI}$ and HA groups. The mean scores for the PCC measure were $68.7 \%$ (standard deviation, $10.5 \%$ ) for the CI group and $69.0 \%$ (standard deviation, $10.5 \%$ ) for the HA group.

The effects of time were allowed for with three variables: the age at "onset" of hearing loss, the period of "deafness" between the onset of hearing loss and implantation (CI group only), and the period of "experience" from implantation to the date of evaluation (CI group) or from the onset of hearing loss to the date of evaluation (HA group). The date of hearing aid fitting was not known for some children in the HA group and was assumed to be shortly after the onset of hearing loss. 




Figure 5-1. CNC word scores in the auditory condition versus chronological age for children using a cochlear implant $(\mathrm{Cl})$ or hearing aid (HA). From Blamey et al. (2001). Copyright by the American Speech-Language-Hearing Association. Reprinted with permission.

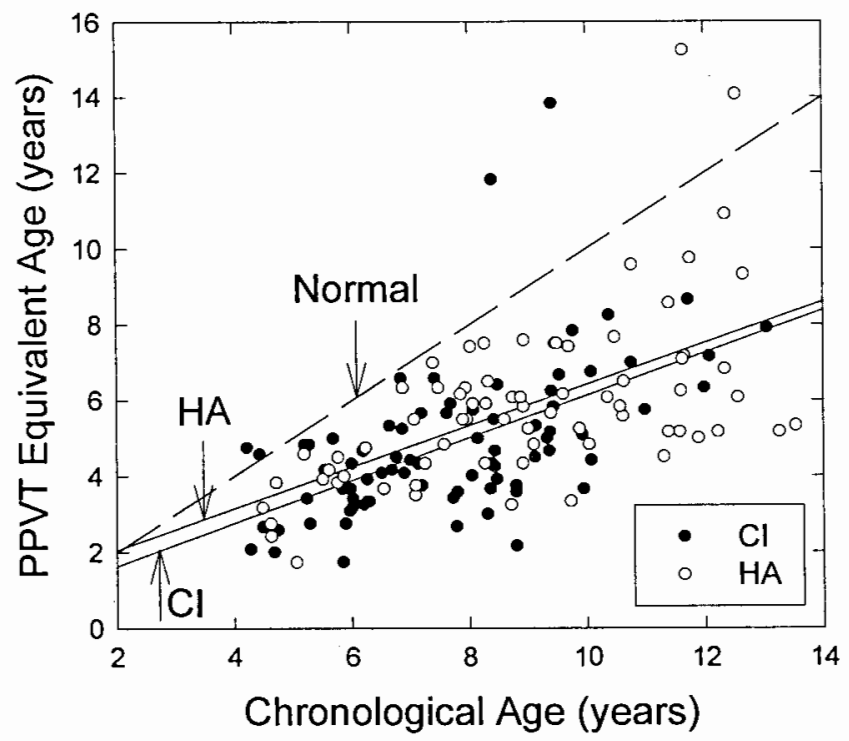

Figure 5-2. PPVT equivalent age versus chronological age for children using a cochlear implant $(\mathrm{Cl})$ or hearing aid $(\mathrm{HA})$. The dashed line indicates average results for typical hearing children. From Blamey et al. (2001). Copyright by the American Speech-Language-Hearing Association. Reprinted with permission. 
Multiple regression analyses were performed for all perception, production, and language measures as dependent variables, with onset, deafness, experience, and PTA hearing loss as independent variables. The regression equations are shown in table 5.2. Each equation shows how children's scores on the various measures changed on average as a function of time. For example, the second equation for the $\mathrm{CI}$ group says that the average score for CNC words in the auditory-alone condition increased by $3.60 \%$ for every year prior to the child's onset of deafness, decreased by $2.37 \%$ for every year after the child became deaf and before the cochlear implant operation, and then increased again by $3.24 \%$ for every year after the operation. This is consistent with the gentle upward trend shown in figure 5.1. The same equation shows that, on average, CNC word scores in the auditory-alone (A) condition were lower by $0.38 \%$ for every decibel of hearing loss.

The effects of the time variables for the PPVT equivalent age analyses were all statistically significant. For example, the PPVT equivalent age for the $\mathrm{CI}$ group increased by 0.67 years for every year of chronological age prior to the onset of deafness, by 0.57 years during the period between onset of deafness and cochlear implantation, and by 0.68 years during the period following the implant operation. This corresponds to strong language growth, as shown in figure 5.2, but unfortunately, the average rate of growth is only about two thirds of the rate for hearing children. This means that, on average, these deaf and hard-of-hearing children were likely to enter secondary school (at about age 12) with vocabulary knowledge equivalent to an 8-year-old hearing child.

Table 5-2: Regression Analyses of Perception and Language Scores as a Function of Age at Onset of Hearing Loss, Duration of Deafness, Experience, and PTA Hearing Loss

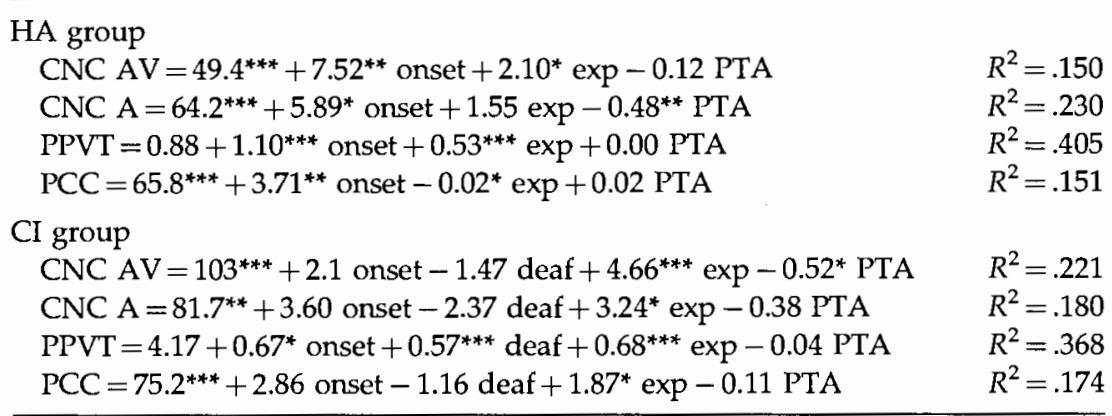

For CNC and PCC, units are percent for the scores and the constant coefficients, percent per $\mathrm{dB}$ HL for PTA, and percent per year for onset, deafness, and experience coefficients. For PPVI, the units are years for the equivalent age and the constant coefficients, and years per $\mathrm{dB}$ HL for PTA. The coefficients for onset, deafness, and experience are dimensionless for the PPVT equations.

${ }^{*} p<.05 ;{ }^{* *} p<.01 ;{ }^{* *} p<.001$. 
It was hoped that the coefficients for the time variables in table 5.2 would all be significantly greater than zero, indicating that the children were improving in speech perception, vocabulary knowledge, and speech production accuracy over time. It was also expected that the coefficients for PTA would be significantly less than zero, indicating that the children with greater hearing loss had lower scores than children with lower hearing thresholds.

As shown by the asterisks in table 5.2, 12 of the 20 time coefficients were statistically significantly greater than zero, and two of the eight PTA coefficients were significantly less than zero. Despite these significant trends in the expected directions, the proportions of variance accounted for by the multiple regression analyses were less than or equal to $40.5 \%$ (see $R^{2}$ values in table 5.2 ). It is likely that much of the remaining variance is related to other variables not included in the regression equations of table 5.2. In the case of the CNC scores, the additional variables include the spoken language abilities of the child, as discussed in the introductory remarks to this chapter. Using the PPVT and PCC measures as additional independent variables in the analyses for CNC scores results in the regression equations of table 5.3. The PPVT equivalent age values used in these regression analyses were limited to a maximum of 7 years because it was found empirically that the perception scores reached a maximum at about this language level (Blamey et al., 2001). The coefficients of PPVT indicate the increase in percent score for each year of equivalent language age up to a maximum of 7 years. For example, the first regression equation indicates

Table 5-3: Regression Equations for Speech Perception Scores as a Function of Time, Hearing Loss, and Language and Speech Production

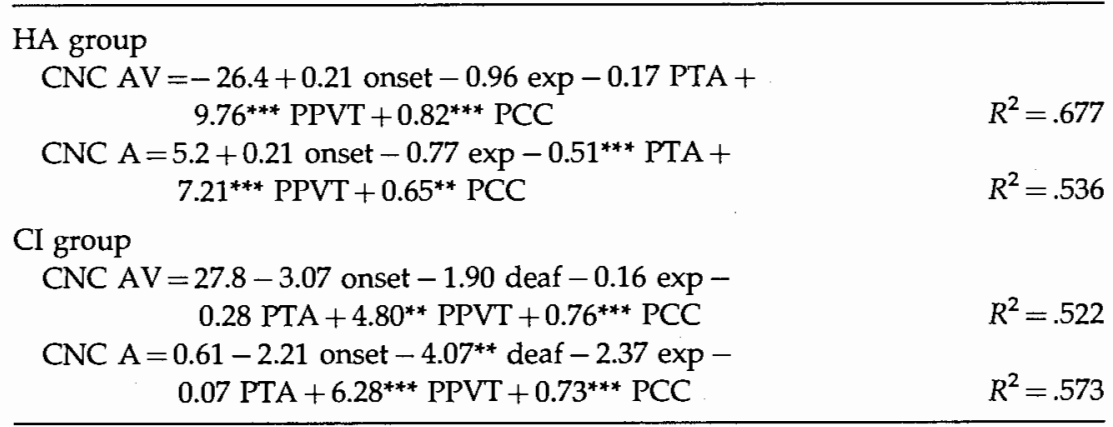

Units are percent for perception scores and constant coefficients; percent per year for onset, deafness, experience, and PPVT coefficients; and percent per dB HL for PTA. Units for the PCC terms are dimensionless because both the perception and production scores are percentages.

${ }^{*} p<.05 ;{ }^{* *} p<.01 ;{ }^{* * *} p<.001$. 
that the average CNC word score in the AV condition increased by $4.80 \%$ for the CI group as the children's equivalent age increased by 1 year. Similarly, the average score increased by $0.76 \%$ as the children's percentage of consonants correctly produced increased by $1 \%$.

Comparison of tables 5.2 and 5.3 shows that the range of variance accounted for in the CNC analyses has increased from $15-23 \%$ in table 5.2 to $52-67 \%$ in table 5.3 when the language-based measures were introduced as additional independent variables. As expected, the coefficients for the PPVT and PCC measures are all significantly greater than zero in table 5.3. None of the 10 time coefficients (for onset, deafness, and experience) is significantly greater than zero. This means that all of the learning that contributed to increases in the CNC perception scores of these children was associated with spoken language improvements. Only one of the PTA coefficients was statistically significantly different from zero, meaning that perception scores were more significantly associated with linguistic performance than with hearing for all except the CNC A scores for the HA group.

No matter how strong an association may be between two measures, the association does not necessarily demonstrate a causal relationship one way or the other. It may be that children who have more hearing have better speech perception and therefore develop spoken language faster, or it may be that children who learn spoken language faster have higher speech perception scores. Whether the relationships are causal is of more than academic interest. If better hearing allows spoken language to develop faster, then children with hearing loss should be fitted with hearing aids or cochlear implants as early as possible. If spoken language performance is a major factor affecting speech perception, then habilitation should be directed at developing spoken language as quickly and effectively as possible, rather than concentrating entirely on improving hearing. It is possible, even probable, that both relationships are causal and better hearing makes it easiertolearn spoken language, and better spoken language yièlds higher spèech perception scores. In this case, when an individual child scores low for speech perception, one needs to know whether their performance is limited by their hearing, or their spoken language, or both, in order to provide the most effective habilitation program. This is the subject of the next section.

\section{SEPARATING CONTRIBUTIONS OF HEARING, LEXICAL KNOWLEDGE, AND SPEECH PRODUCTION TO SPEECH PERCEPTION}

In contrast to the multiple regression analysis in the previous section, the study of Paatsch et al. (2004) used a nonlinear mathematical model to describe the specific effects of hearing, lexical knowledge, and speech 
production on monosyllabic word perception scores. Thirty-three school children with hearing loss, fitted with hearing aids and/or cochlear implants, were evaluated using speech-perception, readingaloud, speech-production, and language measures. These measures were incorporated into the mathematical model, which revealed that the word perception test scores in the auditory-alone mode were strongly dependent on residual hearing levels, lexical knowledge, and speechproduction abilities. This is similar to the result described in the preceding section; however, the mathematical model also provided estimates of the separate effects of hearing and spoken language performance on the overall speech perception score for each child. This information is very important in optimizing the habilitation program for individual children.

Thirty-three children ( 16 boys and 17 girls) between 6 and 14 years of age participated in this study. Twenty of the children had a hearing loss greater than $90 \mathrm{~dB} \mathrm{HL}$, seven had a severe hearing loss (70-89 dB $\mathrm{HL}$ ), and six had a moderate hearing loss (40-69 dB HL). Twenty-one of the children were fitted with behind-the-ear hearing aids by audiologists from Australian Hearing (An Australian government agency providing hearing aids to children throughout Australia). Twelve children were cochlear implant users who were implanted with the Nucleus 22 multichannel device (Clark et al., 1987) using the SPEAK speech processing strategy (Whitford et al., 1995). These children were managed by the Melbourne Cochlear Implant Clinic.

Table 5.4 shows the pure tone aided average thresholds at 500, 1000, and $2000 \mathrm{~Hz}$ for the better ear from the most recent audiogram, and the age for each child. None of the children in the study had an uncorrected visual impairment or known sensory dyslexia. During the selection process, one child was excluded for this reason. Children with very low reading levels (i.e., children who would have required assistance from the tester to read the monosyllabic words) were also excluded from the study. All 33 children attended mainstream primary and/or secondary schools where an oral/aural method of communication was used. Each school provided additional support for these children during mainstream classroom activities and within small group and/or individual sessions.

This study used evaluations of speech perception and spoken language that were very similar to those in the longitudinal study described above. Two lists of the CNC word test (Peterson \& Lehiste, 1962) were presented to each child in the auditory-alone condition (listening without lipreading). Each participant also was required to read the two lists of $\mathrm{CNC}$ words presented in the auditory-alone condition plus an additional two lists. All CNC responses were videotaped, transcribed, and scored using the criteria that all three phonemes must be correctly produced for the word to be correct. A broad phonemic transcription 
Table 5-4: Individual Scores for Percent Correct CNC Words in the Auditory Alone $(A)$ and Read (R) Conditions $(n=3)$

\begin{tabular}{|c|c|c|c|c|c|c|}
\hline Participant & $\begin{array}{l}\text { Age } \\
\text { (yr:mo) }\end{array}$ & $\begin{array}{l}\text { PTA } \\
\text { (dB HL) }\end{array}$ & $\begin{array}{l}\text { A Word } \\
\text { Scores }\end{array}$ & $\begin{array}{l}\text { R Word } \\
\text { Scores }\end{array}$ & $\begin{array}{l}\text { PPVT } \\
\text { Age-Equivalent } \\
\text { Score (yr:mo) }\end{array}$ & PCC \\
\hline \multicolumn{7}{|c|}{ Cochlear implant users } \\
\hline 1 & $6: 10$ & 107 & 38 & 51 & $5: 3$ & 73 \\
\hline 2 & $7: 8$ & 110 & 37 & 64 & $5: 11$ & 78 \\
\hline 3 & $9: 0$ & 93 & 54 & 96 & $7: 5$ & 85 \\
\hline 4 & $9: 1$ & 115 & 68 & 91 & $7: 3$ & 80 \\
\hline 5 & $10: 0$ & 110 & 45 & 90 & $6: 9$ & 88 \\
\hline 6 & $10: 3$ & 100 & 62 & 78 & $6: 6$ & 85 \\
\hline 7 & $10: 4$ & 105 & 60 & 81 & $5: 10$ & 84 \\
\hline 8 & $10: 5$ & 125 & 48 & 69 & $5: 3$ & 71 \\
\hline 9 & $11: 1$ & 105 & 49 & 81 & $5: 11$ & 78 \\
\hline 10 & $11: 8$ & 113 & 70 & 92 & $8: 8$ & 87 \\
\hline 11 & $13: 0$ & 120 & 53 & 72 & $7: 5$ & 73 \\
\hline 12 & $14: 0$ & 108 & 35 & 67 & $7: 11$ & 65 \\
\hline \multicolumn{7}{|c|}{ Hearing aid users } \\
\hline 13 & $7: 6$ & 97 & 20 & 57 & $4: 10$ & 84 \\
\hline 14 & $8: 8$ & 60 & 70 & 98 & $7: 7$ & 92 \\
\hline 15 & $8: 10$ & 85 & 50 & 84 & $6: 1$ & 84 \\
\hline 16 & $8: 11$ & 45 & 72 & 75 & $7: 7$ & 78 \\
\hline 17 & $8: 11$ & 90 & 13 & 77 & $5: 3$ & 77 \\
\hline 18 & $9: 4$ & 62 & 84 & 97 & 7:11 & 89 \\
\hline 19 & $9: 10$ & 68 & 37 & 94 & $6: 11$ & 84 \\
\hline 20 & $10: 0$ & 83 & 89 & 99 & $7: 6$ & 92 \\
\hline 21 & $10: 6$ & 102 & 24 & 76 & $5: 10$ & 75 \\
\hline 22 & $10: 10$ & 88 & 47 & 56 & $6: 1$ & 73 \\
\hline 23 & $11: 0$ & 68 & 76 & 88 & $5: 9$ & 91 \\
\hline 24 & $11: 4$ & 102 & 25 & 50 & $6: 1$ & 75 \\
\hline 25 & $11: 5$ & 88 & 27 & 73 & $8: 3$ & 79 \\
\hline 26 & $12: 8$ & 103 & 23 & 93 & $8: 2$ & 84 \\
\hline 27 & $12: 8$ & 70 & 76 & 87 & $10: 7$ & 87 \\
\hline 28 & $12: 10$ & 97 & 23 & 51 & $5: 8$ & 74 \\
\hline 29 & $13: 2$ & 75 & 24 & 37 & $5: 2$ & 74 \\
\hline 30 & $13: 4$ & 40 & 55 & 88 & 9:1 & 76 \\
\hline 31 & $13: 5$ & 90 & 29 & 97 & $14: 9$ & 84 \\
\hline 32 & $13: 6$ & 92 & 23 & 90 & $5: 4$ & 78 \\
\hline 33 & $13: 7$ & 78 & 71 & 97 & $8: 10$ & 82 \\
\hline
\end{tabular}

was used in which each phone was transcribed as the nearest English phoneme without diacritics. Interlist variability was assessed by comparing the scores for the pairs of CNC word lists in each condition for each child. The standard deviation of the paired differences across all conditions was $5.45 \%$ for lists of 50 words. Table 5.4 includes the mean 
percentage words correct for the audition (A) and reading $(\mathrm{R})$ conditions for each child.

Conversational speech samples were obtained from each participant by an experienced audiologist or teacher of the deaf. These samples were elicited using prompting questions about familiar topics. All conversations were videotaped. On average, a total of 60 utterances, which were representative of the child's conversational skills, were transcribed phonetically by two linguists. Both linguists had experience in listening to the speech of children with hearing loss. The rules used to separate utterances in these conversation samples included change of speaker, rising and falling intonation (indicating the end of an utterance), a pause of two or more seconds, and/or a single thought constituted as a single utterance. All utterances were transcribed using broad phonetic transcription so that productions of phonemes could be compared with those represented in the CNC words. PCC scores were based on singleton consonants only and excluded unintelligible parts of the conversation. The exclusion of consonants within clusters enabled a comparison of singleton consonants produced in spontaneous conversation with those produced in the elicited $\mathrm{CNC}$ word lists. The total number of phonemes produced by individual participants in the conversation samples ranged from 479 to 1,800 phonemes (mean $=1,050$ ). The total number of words ranged from 200 to 739 words (mean $=437$ ). Individual PCC scores are presented in table 5.4.

The PPVT III (Dunn \& Dunn, 1997) was used to measure each participant's receptive vocabulary. PPVT equivalent age scores for each individual are presented in table 5.4.

The postulated mathematical model allows for three processes that occur when a child performs a word test with a verbal response: the sensory process that transmits phonetic information to the child's mental lexicon, the lexical access process that selects a word from the lexicon, and the production process in which the word is spoken. Different types of error may occur in each process. For example, incomplete or incorrect phonetic information could be heard, the word may be unknown or incorrectly stored in the lexicon, or the child may not have acquired the ability to say all of the phonemes in the word.

. The model assumes that the probability of correctly completing each process is independent of the others. Thus, the probability of making a correct response can be expressed as the product of three probabilities representing correct processing of sensory information, correct processing of lexical information, and correct production of the word:

$$
P_{\mathrm{T}}=P_{\mathrm{S}} \times P_{\mathrm{L}} \times P_{\mathrm{P}}
$$

$P_{\mathrm{T}}$ is the total probability of a correct response, $P_{\mathrm{S}}$ is the probability of correct sensory information being transmitted to the lexicon, $P_{\mathrm{L}}$ is the probability of correctly identifying the word in the lexicon, and $P_{\mathrm{P}}$ is 
the probability of correct production of all phonemes. The model can be applied equally well to reading aloud or to auditory speech perception testing. There is a version of equation 5.1 for each sensory condition measured in the study:

$$
\begin{array}{ll}
P_{\mathrm{TA}}=P_{\mathrm{SA}} \times P_{\mathrm{L}} \times P_{\mathrm{P}} & (A=\text { auditory alone }) \\
P_{\mathrm{TR}}=P_{\mathrm{SR}} \times P_{\mathrm{L}} \times P_{\mathrm{P}} & (R=\text { reading })
\end{array}
$$

$P_{\mathrm{TA}}$ and $P_{\mathrm{SA}}$ are the total probability of a correct response and the probability of correct auditory information being transmitted to the lexicon in the auditory-alone condition, respectively. $P_{\mathrm{TR}}$ and $P_{\mathrm{SR}}$ are the total probability of a correct response and the probability of correct visual information being transmitted to the lexicon in the reading condition, respectively. Because of the visual acuity selection criteria for the participants, it was assumed that there were no sensory errors in the reading condition so that $P_{\mathrm{SR}}=1$. The probabilities of lexical correctness $\left(P_{\mathrm{L}}\right)$ and speech production correctness $\left(P_{\mathrm{P}}\right)$ were hypothesized to be the same in the $A$ and $R$ conditions.

Before the model was applied clinically, the assumptions and predictions of the model were tested rigorously using experimental data (Paatsch et al., 2004). In the interests of brevity and clarity, only the main points of the validation are covered in the following paragraphs. The validation of the model required the use of mathematically transformed data in order to cope with the nonlinear relationships between the variables; however, these transformations are not required for the clinical application of the model that will be described below. The statistical tool chosen to validate the model was multiple factor linear regression. This type of analysis requires a linear additive model rather than the nonlinear multiplicative one in equations 5.1-5.3. In order to separate the multiplicative terms in equations 5.2 and 5.3 into additive terms for the multiple linear regression, a logarithmic transform was applied to the data, as exemplified by the following equation for the auditory speech perception condition:

$$
\begin{aligned}
\log P_{\mathrm{TA}} & =\log \left(P_{\mathrm{SA}} \times P_{\mathrm{L}} \times P_{\mathrm{P}}\right) \\
& =\log P_{\mathrm{SA}}+\log P_{\mathrm{L}}+\log P_{\mathrm{P}}
\end{aligned}
$$

The transformed model predicts that the linear regression analysis of $\log P_{\mathrm{TA}}$ (logarithmically transformed total scores in the A condition) should have significant factors related to hearing $\left(\log P_{\mathrm{SA}}\right)$, lexical measures $\left(\log P_{\mathrm{L}}\right)$, and speech production measures $\left(\log P_{\mathrm{P}}\right)$. Similarly, the analysis of $\log P_{\mathrm{TR}}$ (logarithmically transformed total scores in the $\mathrm{R}$ condition) should have significant factors related to lexical measures $\left(\log P_{\mathrm{L}}\right)$ and speech production measures $\left(\log P_{\mathrm{P}}\right)$ but not to sensory measures. It should be noted that the hypothesis that $P_{\mathrm{SR}}=1$ in the multiplicative model is equivalent to the hypothesis that $\log P_{\mathrm{SR}}=0$ in 
the transformed model. Furthermore, the regression coefficients for the lexical and speech production measures should be the same in the analyses of $\log P_{\mathrm{TA}}$ and $\log P_{\mathrm{TR}}$ because the terms $\log P_{\mathrm{L}}$ and $\log P_{\mathrm{P}}$ are common to both.

To assess these predictions, the measure of hearing ability was the PTA threshold, the lexical measure was PPVT equivalent age, and the production measure was PCC. These measures were used as the independent variables in multiple regression analyses where $\log P_{\mathrm{TA}}$ and $\log P_{\mathrm{TR}}$ were the dependent variables. Unlike the analyses in tables 5.2 and 5.3, the present analyses included children using hearing aids and children using implants within the same group, and thus a comparable measure of hearing was required for both devices. The concept of an equivalent hearing loss for cochlear implant users was introduced by Boothroyd and Eran (1994). An equivalent PTA for the cochlear implant users was derived from the results of Blamey et al. (2001), who reported that a group of 47 children using cochlear implants performed similarly on a broad range of speech perception and language measures to a group of 40 children with an average PTA of $78 \mathrm{~dB}$ HL using hearing aids. The equivalent PTA value of $78 \mathrm{~dB} \mathrm{HL}$ takes into account the average improvement in hearing provided by the cochlear implant. As in the longitudinal study, the PPVT equivalent age was limited to 7 years because speech perception scores in the longitudinal study were not observed to increase further once this level was reached. Log PCC was used as the production measure, with the logarithmic transformation introduced to match the $\log P_{\mathrm{P}}$ term in equation 5.4.

The top two sections of table 5.5 show the results of the linear regression analyses. All of the predictions of the model are satisfied by the experimental data. For the analysis of $\log P_{\mathrm{TA}}$, the regression coefficients corresponding to $\log$ PCC and PTA have values that are statistically significantly different from zero, and the PPVT coefficient is close to significance. For the analysis of $\log P_{\mathrm{TR}}$, the PPVT and $\log$ PCC factors have coefficients that are statistically significantly greater than zero, and PTA has a coefficient that is not statistically significantly different from zero. This pattern of significant factors indicates that auditory speech perception scores are associated with both hearing levels and spoken language performance as expected, and that reading scores do not depend on hearing levels. Comparing the analyses for $\log P_{\mathrm{TA}}$ and $\log P_{\mathrm{TR}}$, the coefficients for PPVT and $\log$ PCC are within one standard deviation of one another, confirming that the lexical and production processes are the same in the auditory speech perception and reading conditions. Thus, the experimental results were consistent with the underlying assumptions of the mathematical model.

Having demonstrated the validity of the mathematical model experimentally, it can be used for its intended purpose, which is to separate the contributions of hearing and spoken language in the $\mathrm{CNC}$ 
Table 5-5: Regression Analyses of Word Scores as a Function of PTA, PPVT, and $\log$ PCC

\begin{tabular}{lccc}
\hline & PTA & PPVT & Log PCC \\
\hline Log $P_{\mathrm{TA}}$ & & & \\
Coefficient & -.008 & .079 & .920 \\
Standard deviation & .002 & .040 & .177 \\
$t$ & -4.14 & 1.99 & 5.20 \\
$p$ & $.001^{*}$ & .055 & $.001^{*}$ \\
Log P & & & \\
Coefficient & -.00042 & .067 & .783 \\
Standard deviation & .001 & .020 & .089 \\
$t$ & -0.45 & 3.36 & 8.76 \\
$p$ & .659 & $.002^{*}$ & $.001^{*}$ \\
Log P & & & \\
Coefficient & -.0074 & .012 & .137 \\
Standard deviation & .0020 & .042 & .189 \\
$t$ & -3.67 & 0.28 & 0.73 \\
$p$ & $.001^{*}$ & .780 & .472 \\
\hline
\end{tabular}

*Significant $p$-value.

word test scores for individual children. Dividing equation 5.2 by equation 5.3 and setting $P_{\mathrm{SR}}=1$ yields an estimate of the auditoryalone sensory contribution that has been separated from the linguistic processing components:

$$
\begin{aligned}
P_{\mathrm{SA}} & =\left(P_{\mathrm{SA}} \times P_{\mathrm{L}} \times P_{\mathrm{P}}\right) /\left(P_{\mathrm{L}} \times P_{\mathrm{P}}\right) \\
& =P_{\mathrm{TA}} / P_{\mathrm{TR}}
\end{aligned}
$$

Equation 5.5 was used to calculate the percentage of hearing errors $\left(P_{\mathrm{SA}}\right)$ for each child from the data in table 5.4. This percentage is shown by the lighter gray portions of the bars in figure 5.3. The percentage of words correct in the auditory condition is shown by the darker gray portions of the bars. The central black portions of the bars represent the percentage of spoken language errors (lexical and production errors). The children with cochlear implants are to the left of the break in the graph, and the hearing aid users are to the right of the break. The hearing aid users have been sorted in order of increasing hearing loss from left to right.

As an additional check on the model, the logarithmically transformed $P_{\mathrm{SA}}$ values were subjected to a linear regression analysis, and the results are shown in the bottom section of table 5.5. As the model predicts, the coefficient for the hearing factor (PTA) is significantly different from zero, and the coefficients for PPVT and log PCC are not significantly different from zero. Thus, the model has been successful in separating the hearing component from the spoken language components. 


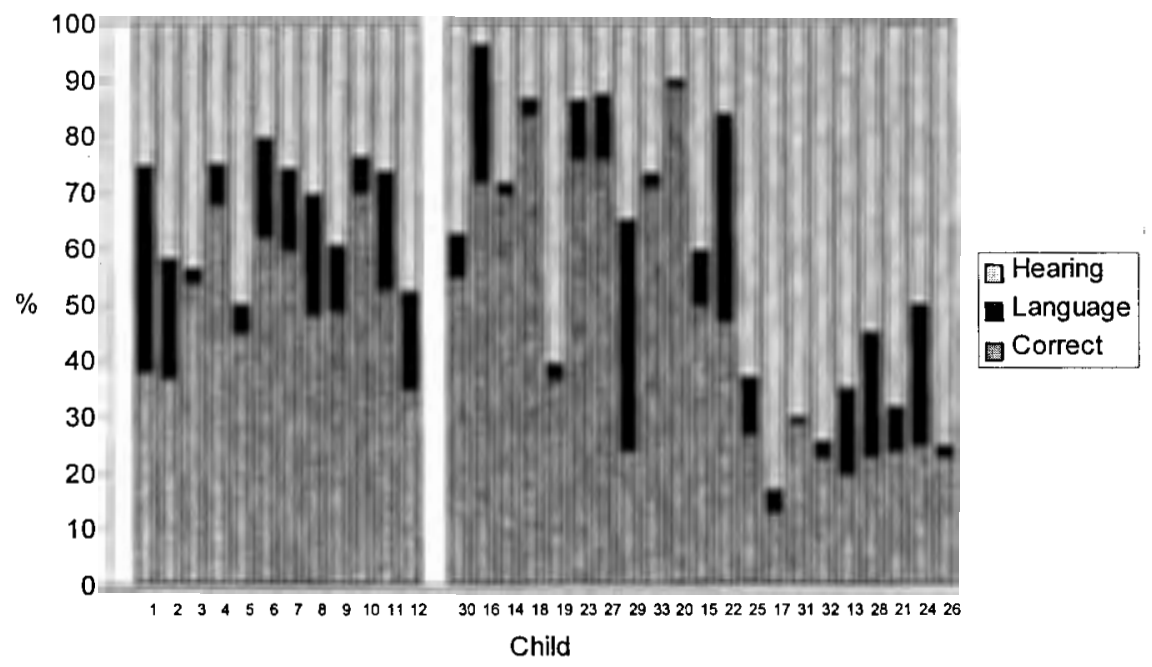

Figure 5:3. CNC word test results for individual children, partitioned into words correct, hearing errors, and spoken language errors.

Inspection of figure 5.3 shows that four children $(1,16,22$, and 29) made more language errors than hearing errors. These children will benefit most from language-based habilitation. Twenty-three of the children made language errors on $10 \%$ or more of the read words. These children would also benefit from language-based habilitation. Prior to the commencement of language-based habilitation, a complete psychological and language assessment might be appropriate to determine whether the child has more general learning difficulties and/or a specific language impairment in addition to their hearing loss.

The sensory score $\left(P_{\mathrm{SA}}\right)$ for the CI users averaged $66.6 \%$ with a standard deviation of $10.5 \%$. Eight of the nine hearing aid users with the greatest hearing loss (greater than $88 \mathrm{~dB} \mathrm{HL}$ ) have sensory scores two standard deviations or more below the CI mean. These children $(13,17$, $21,25,26,28,31$, and 32 ) are likely to benefit most from using a cochlear implant instead of their hearing aids. Child 24 is a little less likely to benefit from a cochlear implant because her sensory score is already about the same as the lower range of sensory scores for the CI group. Note that there appears to be a fairly sudden drop in the sensory scores for hearing aid users at around $88 \mathrm{dBHL}$, separating the listeners with relatively good scores from those with relatively low scores. This boundary is close to the critical level of hearing separating deaf from hard-of-hearing children found in the longitudinal study data (Blamey, Paatsch, Bow, Sarant, \& Wales, 2002).

One HA user stands out as different from the rest. Given his hearing loss of $68 \mathrm{dBHL}$ and his relatively good spoken language performance, 
child 19 performed exceptionally poorly on the CNC word test in the auditory condition. There are several possible explanations for child 19 's level of performance. It is possible that he would benefit from an improved hearing aid fitting. It is also possible that he has a condition known as auditory neuropathy that degrades the quality of hearing much more than would be expected from the audiometric thresholds (Rance, Cone-Wesson, Wunderlich, \& Dowell, 2002), or that he has a fluctuating hearing loss, or the hearing aid was not working correctly on the day of the testing. The recommended response to this result would be an audiological assessment and hearing aid evaluation.

In addition to the diagnostic function illustrated above, the model may be useful in understanding the effects of specific habilitation methods on speech perception scores. The authors speculate that the hearing component is determined primarily by the hearing loss in combination with the assistive devices used and is not easily increased by training. If this is so, then auditory training will be effective only if it produces improvements in the lexical and/or production components. This is more likely to occur if the training is done using words in a meaningful context instead of nonsense syllables. Paatsch, Blamey, and Sarant (2001) found a similar effect in that phoneme production training was more effective in a meaningful context than in a nonmeaningful context. Further studies have found improvements in speech perception as a consequence of training the meanings of words (Sarant, Blamey, Bow, \& Paatsch, 2004) and training of phonology and morphology (Bow, Blamey, Paatsch, \& Sarant, 2004). These training studies support the causal nature of the association between language performance and speech perception scores. It remains to be shown that the improvements observed in speech perception were entirely due to increases in the linguistic terms and not in the sensory term of the detailed model. The model would also need to be elaborated further if it was to include the effects of morphology in addition to lexical knowledge and consonant production.

\section{CONCLUSION}

This chapter has outlined the empirical evidence supporting the need for specific language-oriented habilitation for deaf and hard-of-hearing children, whether they are using cochlear implants or hearing aids. Quantitative methods for assessing the effectiveness of the habilitation procedures and devices have also been outlined. These methods allow the effects of hearing abilities and devices to be separated from the effects of language performance. This is an essential step in optimizing the effectiveness of habilitation for deaf children in general and for individuals who may have specific needs that are different from the general population. 


\section{REFERENCES}

Blamey, P. J. (2002). Development of spoken language by deaf children. In M. Marschark \& P. Spencer (Eds.), Oxford handbook of deaf studies, language, and education (pp. 232-246). New York: Oxford University Press.

Blamey, P. J., Paatsch, L. E., Bow, C. P., Sarant, J. Z., \& Wales, R. J. (2002). A critical level of hearing for speech perception in children. Acoustics Research Letters Online, 3, 18-23.

Blamey, P. J., Sarant, J. Z., Paatsch, L. E., Barry, J. G., Bow, C. P., Wales, R. J., et al. (2001). Relationships among speech perception, production, language, hearing loss, and age in children with impaired hearing. Journal of Speech, Language, and Hearing Research, 44, 264-285.

Boothroyd, A., \& Eran, O. (1994). Auditory speech perception capacity of child implant users expressed as equivalent hearing loss. The Volta Review, 96(5), 151-167.

Bow, C. P., Blamey, P. J., Paatsch, L. E., \& Sarant, J. Z. (2004). The effects of phonological and morphological training on speech perception scores and grammatical judgements in children with impaired hearing. Journal of Deaf Studies and Deaf Education, 9, 305-314.

Brown, R (1973). A first language: The early stages. Cambridge, MA: Harvard University Press.

Clark, G. M., Blamey, P. J., Brown, A. M., Busby, P. A., Dowell, R. C., Franz, B. K.-H., Pyman, B. C., Shepherd, R. K., Tong, Y. C., Webb, R. L., Hirshonn, M. S., Kuzma, J., Mecklenburg, D. J., Money, D. K., Patrick, J. F., \& Seligman, P. M. (1987). The University of Melbourne-Nucleus multi-electrode cochlear implant. Advances in Oto-Rhino-Laryngology, 38, 1-189.

Cutler, A., \& Swinney, D. A. (1987). Prosody and the development of comprehension. Journal of Child Language, 14(1), 145-167.

Dunn, L. M., \& Dunn, L. M. (1981). Peabody Picture Vocabulary test-revised. Circle Pines, MN: American Guidance Service.

Dunn, L. M., \& Dunn, L. M. (1997). Peabody Picture Vocabulary test (3rd ed.). Circle Pines, MN: American Guidance Service.

Eimas, P. D., \& Corbit, J. D. (1973). Selective adaptation of linguistic feature detectors. Cognitive Psychology, 4, 99-109.

Jusczyk, P. (1993). How word recognition may evolve from infant speech perception capacities. In G. T. M. Altmann \& R. Shillcock (Eds.), Cognitive models of speech processing: The second Sperlonga meeting (pp. 27-56). Hove: Lawrence Erlbaum.

Klatt, D. H. (1979). Speech perception: A model of acoustic-phonetic analysis and lexical access. Journal of Phonetics, 7, 279-312.

Mehler, J., Jusczyk, P., Lambertz, G., Halsted, N., Bertoncini, J., \& Amiel-Tison, C. (1988). A precursor of language acquisition in young infants. Cognition, 29, 143-178.

Paatsch, L. E., Blamey, P. J., \& Sarant, J. Z. (2001). Effects of articulation training on the production of trained and untrained phonemes in conversations and formal tests. Journal of Deaf Studies and Deaf Education, 6(1), 32-42.

Paatsch, L. E., Blamey, P. J., Sarant, J. Z., Martin, L. F. A., \& Bow, C. P. (2004). Separating contributions of hearing, lexical knowledge and speech 
production to speech perception scores in children with hearing impairments. Journal of Speech, Language, Hearing Research, 47(4), 738-750.

Peterson, G. E., \& Lehiste, I. (1962). Revised CNC lists for auditory tests. Journal of Speech and Hearing Disorders, 27, 62-70.

Rance, G., Cone-Wesson, B., Wunderlich, J., \& Dowell, R. (2002). Speech perception and cortical event related potentials in children with auditory neuropathy. Ear and Hearing, 23, 239-253.

Sarant, J. Z., Blamey, P. J., Bow, C. P., \& Paatsch, L. E. (2004). Effects of vocabulary training on word knowledge and perception. Manuscript submitted for publication.

Semel, E., Wiig, E., \& Secord, W. A. (1995). Clinical evaluation of language fundamentals (3rd ed.). San Antonio, TX: Psychological Corporation, Harcourt Brace.

Shriberg, L. D., Austin, D., Lewis, B. A., Sweeny, J. L., \& Wilson, D. L. (1997). The percentage of consonants correct (PCC) metric: Extensions and reliability data. Journal of Speech, Language, and Hearing Research, 40, 708-722.

Vihman, M. M. (1996). Phonological development: The origins of language in the child. Cambridge, MA: Blackwell Publishers.

Werker, J. F., \& Tees, R. C. (1984). Cross-language speech perception: Evidence for perceptual reorganization during the first year of life. Infant Behaviour and Development, 7, 49-63.

Whitford, L. A., Seligman, P. M., Everingham, C. E., Antognelli, T., Skok, M. C., Hollow, R. D., Plaut, K. L., Genn, E. S., Staller, S. J., \& McDermott, H. J. et al. (1995). (Evaluation of the Nucleus Spectra 22 processor and new speech processing strategy (SPEAK) in postlinguistically deafened adults. Acta Otolaryngologica, 115(5), 629-637.

Wiig, E., Secord, W. A., \& Semel, E. (1992). Clinical evaluation of language fundamentals-preschool. San Antonio, TX: Psychological Corporation, Harcourt Brace. 


\section{University Library}

\section{- M M I N E R VA A gateway to Melbourne's research publications}

Minerva Access is the Institutional Repository of The University of Melbourne

Author/s:

Blamey, PJ;Sarant, JZ;Paatsch, LE

Title:

Relationships Among Speech Perception and Language Measures in Hard-of-Hearing Children

Date:

2005-09-29

Citation:

Blamey, P. J., Sarant, J. Z. \& Paatsch, L. E. (2005). Relationships Among Speech Perception and Language Measures in Hard-of-Hearing Children. Spencer, P (Ed.). Marschark, M (Ed.). Advances in the Spoken Language Development of Deaf and Hard-of-Hearing Children, (1), pp.85-102. Oxford University Press.

Persistent Link:

http://hdl.handle.net/11343/31495 